\title{
The Reconstruction of the Digital Hyperbola Segment from Its Code
}

\author{
Nataša Sladoje \\ Faculty of Engineering \\ Trg D. Obradovića 6, 21000 NoviSad, Yugoslavia \\ sladoje@uns.ns.ac.yu
}

\begin{abstract}
It is known that a set consisting of the digital curves whose "original curves" are graphs of continuous functions, having at most two intersection points, pairwise, on a given interval, can be uniquely coded by five parameters. This result is applied to the set of digital hyperbola segments, corresponding to the hyperbolas of the form $y=$ $\frac{\alpha}{x-\beta}+\gamma$ inscribed into the $(m \times m)$-integer grid. An $\mathcal{O}\left(m \cdot(\log (m+|\beta|))^{2}\right)$ algorithm for recovering the digital hyperbola segment from its proposed code, is presented.
\end{abstract}

Keywords: image processing, pattern analysis, reconstruction, digital hyperbola.

\section{Introduction}

The most often appearing digital curves in practice are those obtained by digitizing straight lines and conic sections. For the most of them the basic problems considered in computer vision and image processing, such as the efficient representation and reconstruction, as well as the parameter estimation, are already solved, by using different approaches [2]-[7],[10]. The coding scheme developed for the sets of the digital straight line and parabola segments, based on the least squares technique, is successfully applied to the set of the digital hyperbola segments of the form $y=\frac{\alpha}{x}+\beta$ [9]. Unfortunately, that concept, which has the advantage of saving information of the approximate form and position of the original object, could not be applied to the segment of the general hyperbola because, in that case, the determination of the coefficients of the least squares hyperbola fit leads to a nonlinear problem of great complexity. The solution of a coding problem for the general digital hyperbola segment is suggested in [8]. It is shown that the set consisting of the digital curves whose "original curves" are graphs of continuous functions, having at most two intersection points, pairwise, on a given interval, can be uniquely coded by five parameters. This result, specified to the set of the digital hyperbola segments, is briefed in Section 2 and the optimality of the proposed code is considered. One-to-one correspondence established between the digital hyperbola segments and their representations enables 
reconstruction of the digital hyperbola segment from its proposed code. The recovering algorithm is described in Section 3. As an illustration, some numerical results are presented. In Section 4 some concluding remarks are given.

As it is usual in the digital picture analysis, we assume through the paper that all the appearing points have positive coordinates. In other words, the origin is placed in the left-lower corner of the given picture.

\section{Preliminaries}

Consider a hyperbola $h$, in the Euclidean plane, given by $y=\frac{\alpha}{x-\beta}+\gamma$. It is digitized by digitizing method in which the first digital points (points with integer coordinates) below the curve are taken.

We will be dealing with digital hyperbola segment obtained by digitizing part of the hyperbola lying between lines $x=x_{1}$ and $x=x_{2}$ for some $x_{1}$ and $x_{2}$. Without loss of generality we can assume that $x_{1}$ and $x_{2}$ are integers. We also require that $\beta \notin\left[x_{1}, x_{2}\right]$; otherwise, the problem can be divided onto two independent digitization processes: the first one on the interval $\left[x_{1},\lfloor\beta\rfloor\right]$ and the second one on the interval $\left[[\beta], x_{2}\right]$. If $x_{1}=k$ and $x_{2}=k+n-1$, then the associated set of digital points for the segment of the hyperbola $h$ is the digital hyperbola segment $H_{k}(h, n)$, defined by

$$
H_{k}(h, n)=\left\{\left(i,\left\lfloor\frac{\alpha}{i-\beta}+\gamma\right\rfloor\right), i=k, k+1, \ldots, k+n-1\right\},
$$

where $\lfloor t\rfloor$ is the largest integer no bigger than $t$. Then $n$ is the length of the digital hyperbola segment $H_{k}(h, n)$, i.e. the number of digital points of $H_{k}(h, n)$.

So, if we denote $y_{i}=\left\lfloor\frac{\alpha}{i-\beta}+\gamma\right\rfloor$, for $i=k, k+1, \ldots, k+n-1$ and $\beta \notin$ $[k, k+n-1]$, then the digital hyperbola segment $H_{k}(h, n)$ of an arbitrary length, is coded by five parameters $(k, n, a(h), b(h), c(h))$, where:

- $k$ is abscissa of the left endpoint of the digital hyperbola segment $H_{k}(h, n)$;

- $n$ is the length of the digital hyperbola segment $H_{k}(h, n)$;

- $a(h)=\sum_{i=k}^{k+n-1} y_{i}$

- $b(h)=\sum_{i=k}^{k+n-1} i \cdot y_{i}$

- $c(h)=\sum_{i=k}^{k+n-1} g(i) \cdot y_{i}$, where $g(x)$ is an arbitrary strictly convex or strictly concave function having a second derivative on $[k, k+n-1]$.

From the general result proved in [8], it follows that there do not exist two different digital hyperbola segments with the same proposed code. The proof of the statement, specified to the digital segments corresponding to the hyperbolas in the general form, is briefly reported here, since some of the details will be needed in the recovering procedure analysis. As it is already mentioned, the size of the observed grid is denoted by $m$. An auxiliary statement is used: 
Lemma 1 Let a sequence of nonnegative numbers, satisfying

$$
0 \leq a_{1} \leq a_{2} \leq \ldots \leq a_{t}<b_{1} \leq b_{2} \leq \ldots \leq b_{n}<a_{t+1} \leq \ldots \leq a_{n}
$$

and

$$
a_{1}+a_{2}+\ldots+a_{n}=b_{1}+b_{2}+\ldots+b_{n}
$$
and let $g(x)$ be either a strictly convex or a strictly concave function, which has a second derivative on the interval $\left[a_{1}, a_{n}\right]$. Then

$$
g\left(a_{1}\right)+g\left(a_{2}\right)+\ldots+g\left(a_{n}\right) \neq g\left(b_{1}\right)+g\left(b_{2}\right)+\ldots+g\left(b_{n}\right) .
$$

The proof of this statement is also given in [8].

Theorem 1 Let $g(x)$ be a strictly convex (concave) function having a second derivative on the interval $[0, m-1]$ and let $H_{r}(l, p)$ and $H_{s}(t, q)$ be digital hyperbola segments, with $(r, p, a(l), b(l), c(l))$ and $(s, q, a(t), b(t), c(t))$ - codes, respectively. Then

$$
\begin{gathered}
H_{r}(l, p)=H_{s}(t, q) \quad \text { is equivalent to } \\
(r=s \wedge p=q \wedge a(l)=a(t) \wedge b(l)=b(t) \wedge c(l)=c(t)) .
\end{gathered}
$$

Proof. From the definition of the proposed code follows that

$$
\begin{aligned}
& H_{r}(l, p)=H_{s}(t, q) \quad \text { implies } \\
& (r=s \wedge p=q \wedge a(l)=a(t) \wedge b(l)=b(t) \wedge c(l)=c(t)) .
\end{aligned}
$$

The opposite direction will be proved by a contradiction.

For the given digital hyperbola segment $H_{k}(h, n)$, the set $D_{k}(h, n)$ containing all the digital points lying above the $x$-axis and below the hyperbola $h$, with abscissas belong to the interval $[k, k+n-1]$ is observed. Then the interpretation of the parameters $a(h), b(h), c(h)$ can be given as follows:

- $a(h)=\sum_{i=k}^{k+n-1} y_{i}=\sum_{(x, y) \in D_{k}(h, n)} 1$ is the number of the points of $D_{k}(h, n)$;

- $b(h)=\sum_{i=k}^{k+n-1} i \cdot y_{i}=\sum_{(x, y) \in D_{k}(h, n)} x$

is the sum of the $x$-coordinates of the points of $D_{k}(h, n)$;

- $c(h)=\sum_{i=k}^{k+n-1} g(i) \cdot y_{i}=\sum_{(x, y) \in D_{n}(h, k)} g(x)$,

is the sum of the values of the function $g(x)$ in all the points of $D_{k}(h, n)$.

From the assumption that two different digital hyperbola segments $H_{r}(l, p)$ and $H_{s}(t, q)$ have the same representation, we conclude that they are observed on the same interval $[r, r+p-1]=[s, s+q-1]$, and that the relations

$$
D_{r}(l, p) \neq D_{s}(t, q)
$$




$$
a(l)=a(t) \quad, \quad b(l)=b(t) \quad, \quad c(l)=c(t)
$$

are satisfied.

From (1) and the first relation in (2) follows that

$$
\#\left(D_{r}(l, p) \backslash D_{s}(t, q)\right)=\#\left(D_{s}(t, q) \backslash D_{r}(l, p)\right) \neq 0
$$

which means that hyperbolas $l$ and $t$ must have an intersection point $x_{0} \in$ $[r, r+p-1]$. Assuming $b(l)=b(t)$, we have

$$
\sum_{(x, y) \in D_{r}(l, p) \backslash D_{s}(t, q)} x=\sum_{(x, y) \in D_{s}(t, q) \backslash D_{r}(l, p)} x .
$$

But the sets $D_{r}(l, p) \backslash D_{s}(t, q)$ and $D_{s}(t, q) \backslash D_{r}(l, p)$ can be separated by the straight line $x=x_{0}$ in such way that, let's say, $x<x_{0}$ for $x \in D_{r}(l, p) \backslash D_{s}(t, q)$ and $x>x_{0}$ for $x \in D_{s}(t, q) \backslash D_{r}(l, p)$ (2). Contradiction with (3) follows.

From (3) and the assumption $c(l)=c(t)$ follows that

$$
\sum_{(x, y) \in D_{r}(l, p) \backslash D_{s}(t, q)} g(x)=\sum_{(x, y) \in D_{s}(t, q) \backslash D_{r}(l, p)} g(x) .
$$

If we suppose that $l$ and $t$ have two intersection points $x_{1}, x_{2} \in[r, r+p-1]$ $\left(x_{1}<x_{2}\right)$, the sets $D_{r}(l, p) \backslash D_{s}(t, q)$ and $D_{s}(t, q) \backslash D_{r}(l, p)$ are separated by straight lines, $x=x_{1}$ and $x=x_{2}$ in such way that, let's say, $x<x_{1}$ or $x>x_{2}$ for $x \in D_{r}(l, p) \backslash D_{s}(t, q)$ and $x_{1}<x<x_{2}$ for $x \in D_{s}(t, q) \backslash D_{r}(l, p)(2)$.

In other words, taking into account (3) and (4), we have that the abscissas of the points of $D_{r}(l, p) \backslash D_{s}(t, q)$ and $D_{s}(t, q) \backslash D_{r}(l, p)$, are divided into two sets in such way that the conditions of the Lemma 1 are satisfied. But the result following from Lemma 1 is in contradiction to (5).

Since two hyperbola arcs can have at most two intersection points on the observed interval, the proof is finished.

Since for the appropriate choice of $g(x)$ all of its parameters are integers, this code is optimal, which is proved by the next lemma.

Lemma 2 The $(k, n, a(h), b(h), c(h))$-code requires an asymptotically optimal number of bits.

Proof. For coding numbers $k, n, a(h), b(h)$ and $c(h)$ (for $g(x)$ chosen to be, for example, any polynomial function) $\mathcal{O}(\log m)$ bits are required. Since there are at least $m$ different digital hyperbola segments which can be inscribed into the $m \times m$ integer grid, at least $\log m$ bits are required for representing them.

Remark 1.: If $l: y=\frac{\alpha_{l}}{x-\beta_{l}}+\gamma_{l}$ and $t: y=\frac{\alpha_{t}}{x-\beta_{t}}+\gamma_{t}$ are two hyperbolas corresponding to different digital segments on the interval $[r, r+p-1]$, from the proofs of Lemma 1 (conditions assumed) and Theorem 1 it follows: 


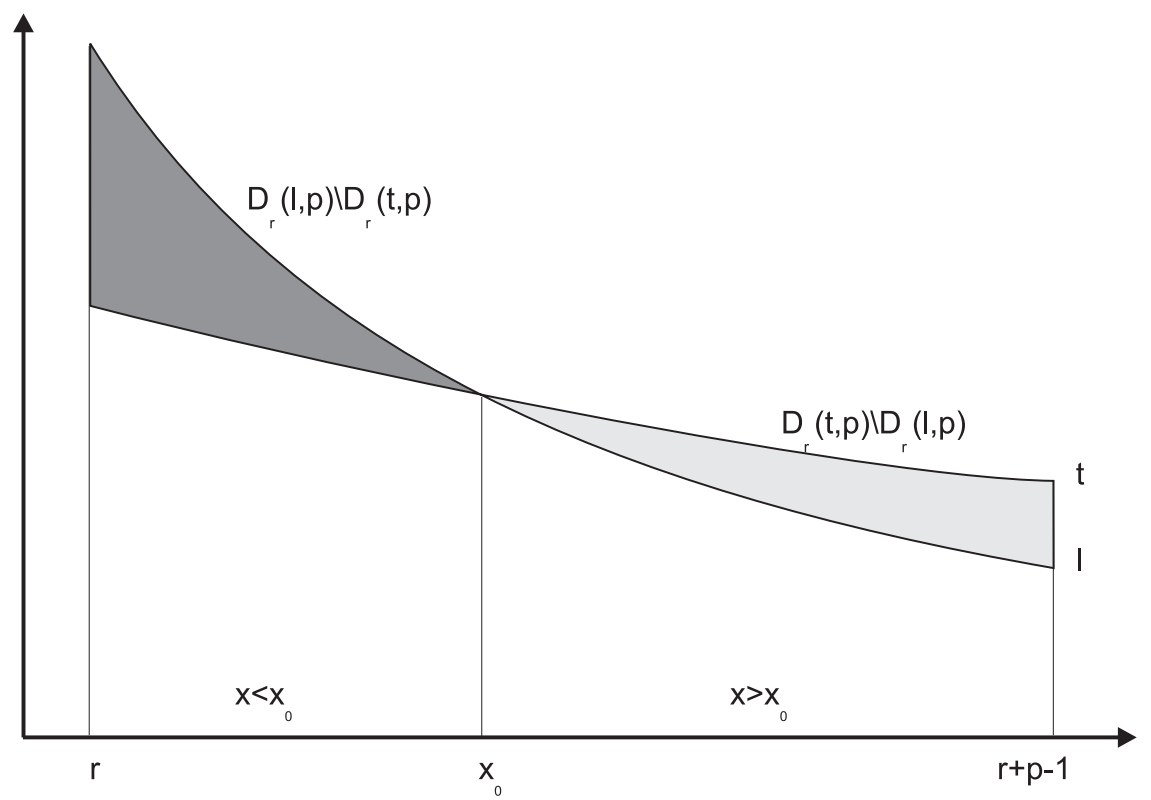

Fig. 1. $l$ and $t$ have one intersection point $x_{0} \in[r, r+p-1]$, so $D_{r}(l, p) \backslash D_{r}(t, p)$ and $D_{r}(t, p) \backslash D_{r}(l, p)$ are separated by $x=x_{0}$. Assuming $a(t)=a(l), b(t)>b(l)$

- If $l$ and $t$ have one intersection point on $[r, r+p-1]$ and $a(l)=a(t)$, then

$$
\frac{\alpha_{l}}{r-\beta_{l}}+\gamma_{l}>\frac{\alpha_{t}}{r-\beta_{t}}+\gamma_{t} \quad \Leftrightarrow \quad b(l)<b(t)
$$

- If $l$ and $t$ have two intersection points on $[r, r+p-1]$ and $a(l)=a(t)$ and $b(l)=b(t)$ hold, then

$$
\begin{aligned}
& \frac{\alpha_{l}}{r-\beta_{l}}+\gamma_{l}>\frac{\alpha_{t}}{r-\beta_{t}}+\gamma_{t} \quad \Leftrightarrow \quad c(l)>c(t) \quad \text { for } \quad g^{\prime \prime}(x)>0 \\
& \frac{\alpha_{l}}{r-\beta_{l}}+\gamma_{l}>\frac{\alpha_{t}}{r-\beta_{t}}+\gamma_{t} \quad \Leftrightarrow \quad c(l)<c(t) \text { for } \quad g^{\prime \prime}(x)<0 .
\end{aligned}
$$

\section{Recovering Algorithm}

The one-to-one correspondence between the set of the digital hyperbola segments and the set of their proposed representations enables reconstruction of the segment from its code. In this section we describe a recovering algorithm.

We assume that $\alpha>0$ and $\beta<k$. Other cases are treated in similar way.

First, we describe a procedure $\operatorname{Set} a(k, n, A, B, a, H)$ which, for a given interval $[k, k+n-1]$, parameters $A, B$ and $a$, finds a hyperbola $H: y=\frac{A}{x-B}+C$, such that $a=a(H)$

Procedure $S e t \_a(k, n, A, B, a, H)$

Input: The numbers $k, n, A, B, a$ 


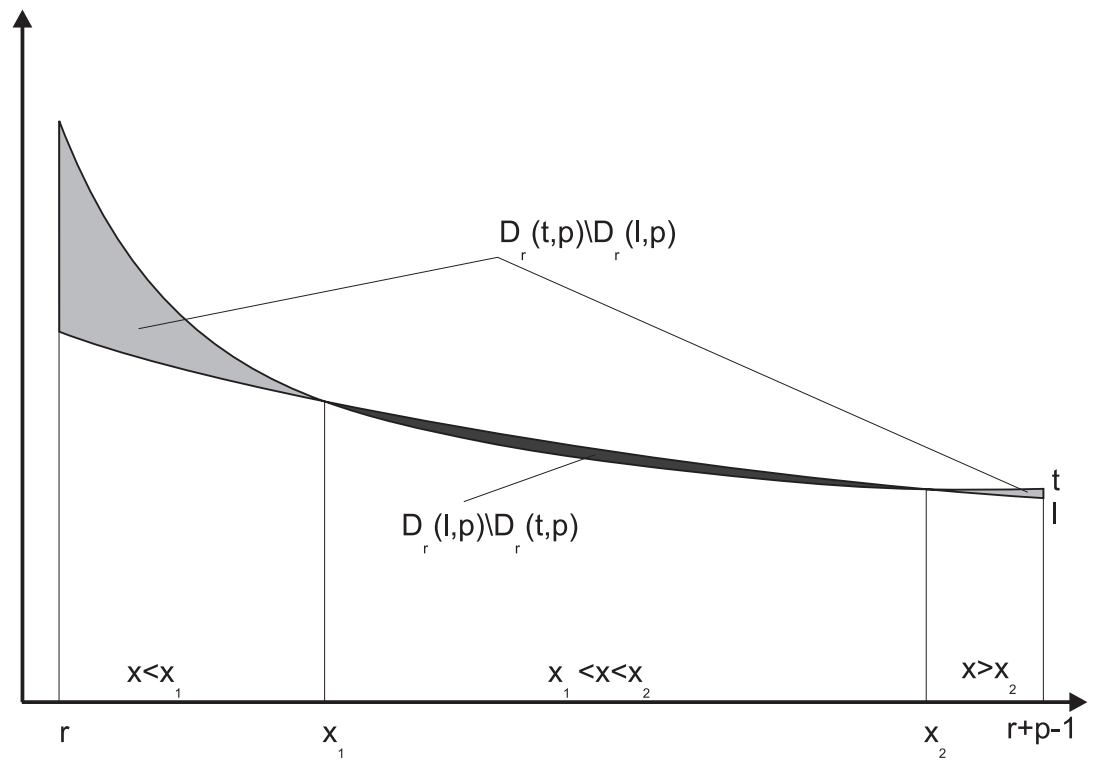

Fig. 2. $l$ and $t$ have two intersection points $x_{1}, x_{2} \in[r, r+p-1]$, and $D_{r}(l, p) \backslash$ $D_{r}(t, p)$ and $D_{r}(t, p) \backslash D_{r}(l, p)$ are separated by $x=x_{1}$ and $x=x_{2}$. Assuming $a(l)=a(t)$ and $b(l)=b(t), c(t)>c(l)$ for $g^{\prime \prime}(x)>0$ and $c(t)<c(l)$ for $g^{\prime \prime}(x)<0$

Output: Hyperbola $H$ of the form $y=\frac{A}{x-B}+C$, satisfying $a(H)=a$.

Step 1: Draw a hyperbola segment $h_{\text {temp }}: y=\frac{A}{x-B}$ and compute $a\left(h_{\text {temp }}\right)$.

Step 2: Translate $h_{t e m p}$ by $\left\lfloor\frac{a-a\left(h_{t e m p}\right)}{n}\right\rfloor$ in the vertical direction (upward if $a-$ $a\left(h_{\text {temp }}\right)>0$ and downward otherwise) and denote obtained hyperbola by $h^{\prime}$. Compute $a\left(h^{\prime}\right)$. (In this way, $\left|a-a\left(h^{\prime}\right)\right|<n$ is preserved).

Step 3: Let $\left(k+i-1, y_{i}\right), i=1,2, \ldots, n$ be the digital points obtained by digitizing the hyperbola $h^{\prime}$. Find the numbers $d_{i}, i=1,2, \ldots, n$, where $d_{i}$ is the vertical distance between the point $\left(k+i-1, y_{i}+1\right), i=1,2, \ldots n$ and the hyperbola $h^{\prime}$.

Step 4: Find the $\left(a-a\left(h^{\prime}\right)\right)$-th smallest number among the numbers $d_{i}$. (Note: Any algorithm that finds the $r$-th median of the set can be used - for example see [1] which is linear time algorithm.)

Step 5: Translate $h^{\prime}$ to its final position $H$ by $\left(a-a\left(h^{\prime}\right)\right)$-th smallest distance, in the vertical direction.

Lemma 3 The time complexity of the procedure Set_a $(k, n, A, B, a, H)$ is $O(m)$.

We continue with the lemma which, for the given coefficient $\beta$, determines the interval $I(\alpha)$ containing coefficient $\alpha$ : 
Lemma 4 If $H_{k}(h, n)=\left\{\left(i, y_{i}\right), i=k, \ldots k+n-1\right\}$ is the digital hyperbola segment obtained by digitizing the hyperbola $h: y=\frac{\alpha}{x-\beta}+\gamma$, then

$$
\alpha \in\left(\frac{2 b(h)-a(h) \cdot(2 k+n-1)+n \cdot(2 k+n-1)}{\sum_{i=1}^{n} \frac{2 i-n-1}{k+i-1-\beta}}, \frac{2 b(h)-a(h) \cdot(2 k+n-1)-n \cdot(2 k+n-1)}{\sum_{i=1}^{n} \frac{2 i-n-1}{k+i-1-\beta}}\right) .
$$

Proof. According to the digitizing method, for $i=1,2, \ldots, n$ it follows:

and

$$
\frac{\alpha}{k+i-1-\beta}+\gamma \in\left[y_{k+i-1}, y_{k+i-1}+1\right)
$$

$$
\frac{\alpha \cdot(k+i-1)}{k+i-1-\beta}+(k+i-1) \cdot \gamma \in\left[(k+i-1) \cdot y_{k+i-1},(k+i-1) \cdot\left(y_{k+i-1}+1\right)\right) .
$$

This implies the following system of inequalities:

$$
\begin{gathered}
\alpha \sum_{i=1}^{n} \frac{1}{k+i-1-\beta}+n \gamma \in[a(h), a(h)+n) \\
\alpha \sum_{i=1}^{n} \frac{k+i-1}{k+i-1-\beta}+\gamma \sum_{i=1}^{n}(k+i-1) \in\left[b(h), b(h)+n k+\frac{n(n-1)}{2}\right) .
\end{gathered}
$$

By solving it, the statement follows. (Note: $\sum_{i=1}^{n} \frac{2 i-n-1}{k+i-1-\beta}<0$.)

Remark 2. From above, it is not difficult to conclude that, for the different values of $\alpha$, while keeping $\beta$ and $a(h)$ fixed, the parameter $b(h)$ can take all the integer values between 1 and $n(2 k+n-1)$.

The following lemma permits a binary searching over the coefficient $\alpha$, while $\beta$ is fixed:

Lemma 5 Let $H^{\prime}$ and $H^{\prime \prime}$ be hyperbolas corresponding to different digital segments, obtained by calling Set_a( $\left(k, n, A^{\prime}, B, a, H^{\prime}\right)$ and Set_a $\left(k, n, A^{\prime \prime}, B, a, H^{\prime \prime}\right)$. Then $A^{\prime}<A^{\prime \prime}$ is equivalent to $b\left(H^{\prime}\right)>b\left(H^{\prime \prime}\right)$.

Proof. Let $H^{\prime}$ and $H^{\prime \prime}$ be given, respectively, by the equations

$$
y=\frac{A^{\prime}}{x-B}+C^{\prime} \quad \text { and } \quad y=\frac{A^{\prime}+\triangle A^{\prime}}{x-B}+C^{\prime \prime}, \quad \text { where } A^{\prime}+\triangle A^{\prime}=A^{\prime \prime} .
$$

From $H_{k}\left(H^{\prime}, n\right) \neq H_{k}\left(H^{\prime \prime}, n\right)$ and $a\left(H^{\prime}\right)=a\left(H^{\prime \prime}\right)=a$ we conclude that the (only) intersection point $x_{0}$ of $H^{\prime}$ and $H^{\prime \prime}$ is in $x_{0} \in(k, k+n-1)$, and that the relation $\left(C^{\prime}-C^{\prime \prime}\right)\left(x_{0}-B\right)=\triangle A^{\prime} \quad$ holds. Since $B<k$ and $x_{0}>k$, we have $\left(x_{0}-B\right)>0$. According to the assumption that $A^{\prime}>0$ and $A^{\prime \prime}>0$, the condition $b\left(H^{\prime}\right)>b\left(H^{\prime \prime}\right)$ is equivalent to $C^{\prime}>C^{\prime \prime}$ (see Remark 1. and Fig.1).

So, we have $b\left(H^{\prime}\right)>b\left(H^{\prime \prime}\right) \Leftrightarrow \triangle A^{\prime}>0 \Leftrightarrow A^{\prime}<A^{\prime \prime}$.

There is a possibility that the hyperbola $H$ of the form $y=\frac{A}{x-B}+C$, satisfying $a=a(H)$ and $b=b(H)$, for the chosen $B$ is not found, which means 
that $|b(H)-b|$ is unreachable for any $A$. It could happen only if the parameter $\beta$, locating the vertical asymptote of the original (digitized) curve is very close to (far from) $k$ (the beginning of the segment). So, in that case, in order to increase (decrease) $b(H)$, the chosen $B$ should be increased (decreased).

Now, we give the procedure which, for a given parameter $B$, interval $[k, k+n-1]$ and numbers $a$ and $b$, finds a hyperbola $H$ of the form $y=\frac{A}{x-B}+C$, such that $a(H)=a$ and $b(H)=b$. (We mention again that $m$ is the grid size).

Procedure Set_ab $(k, n, B, a, b, H)$

Input: The numbers $k, n, B, a, b$.

Output: Hyperbola $H$ of the form $y=\frac{A}{x-B}+C$, with $a(H)=a$ and $b(H)=b$.

Step 1: Set $I(\alpha)=\left(\frac{2 b-a \cdot(2 k+n-1)+n \cdot(2 k+n-1)}{\sum_{i=1}^{n} \frac{2 i n-1}{k+i-1-B}}, \frac{2 b-a \cdot(2 k+n-1)-n \cdot(2 k+n-1)}{\sum_{i=1}^{n} \frac{2 i-n-1}{k+i-1-B}}\right)$;

Choose a coefficient $A \in I(\alpha)$;

Step 2: Set_a $(k, n, A, B, a, H)$;

Step 2: WHILE $(b(H)) \neq b$ and the weight of $\left.I(\alpha)>\frac{1}{16 m^{8}}\right)$ DO

\{chose the next $A \in I(\alpha)$ by using binary searching; $\quad$ (* Lemma $5 *$ )

Set_a $(k, n, A, B, a, H)\}$;

Step 3: IF $(b(H) \neq b)$

(* required accuracy is reached without finding appropriate $A *$ )

\{increase $B$ if $b>b(H)$ or decrease $B$ if $b<b(H)$;

Set_ab $(k, n, B, a, b, H)\}$.

As a result of executing the Procedure $\operatorname{Set} \_a b(k, n, B, a, b, H)$, we have the segment $\left\{\left(i, y_{i}\right), i=k, \ldots, k+n-1\right\}$ of the hyperbola $H: y=\frac{A}{x-B}+C$ for which $a(H)=a$ and $b(H)=b$. If $c(H)=c$, the recovering process is finished. If not, we need the following result which permits binary searching over both of the coefficients $\alpha$ and $\beta$ :

Lemma 6 Let $H^{\prime}$ and $H^{\prime \prime}$ be defined by $y=\frac{A^{\prime}}{x-B^{\prime}}+C^{\prime}$ and $y=\frac{A^{\prime}+\triangle A^{\prime}}{x-\left(B^{\prime}+\triangle B^{\prime}\right)}+$ $C^{\prime \prime}$, respectively and correspond to different digital segments, obtained by calling $\operatorname{Set} a b\left(k, n, B^{\prime}, a, b, H^{\prime}\right)$ and $\operatorname{Set}_{-} a b\left(k, n, B^{\prime \prime}, a, b, H^{\prime \prime}\right)$.

Then $c\left(H^{\prime}\right)>c\left(H^{\prime \prime}\right) \Leftrightarrow\left(A^{\prime}<A^{\prime \prime} \quad\right.$ and $\left.B^{\prime}>B^{\prime \prime}\right) \quad$ if $\quad g^{\prime \prime}(x)>0$,

and $c\left(H^{\prime}\right)>c\left(H^{\prime \prime}\right) \Leftrightarrow\left(A^{\prime}>A^{\prime \prime} \quad\right.$ and $\left.\quad B^{\prime}<B^{\prime \prime}\right) \quad$ if $\quad g^{\prime \prime}(x)<0$.

Proof. From

$$
H_{k}\left(H^{\prime}, n\right) \neq H_{k}\left(H^{\prime \prime}, n\right), \quad a\left(H^{\prime}\right)=a\left(H^{\prime \prime}\right)=a, \quad b\left(H^{\prime}\right)=b\left(H^{\prime \prime}\right)=b,
$$

we conclude that $H^{\prime}$ and $H^{\prime \prime}$ have two intersection points, $x_{1}, x_{2} \in(k, k+n-1)$, satisfying $\left(C^{\prime}-C^{\prime \prime}\right)\left(x-B^{\prime}\right)^{2}-\left(\triangle B^{\prime}\left(C^{\prime}-C^{\prime \prime}\right)+\triangle A^{\prime}\right)\left(x-B^{\prime}\right)-A^{\prime} \triangle B^{\prime}=0$. According to the Remark 1, (see Fig.2) we have $c\left(H^{\prime}\right)>c\left(H^{\prime \prime}\right) \Leftrightarrow C^{\prime}>C^{\prime \prime}$, if $g^{\prime \prime}(x)>0$ on $[k, k+n-1]$, and

$$
c\left(H^{\prime}\right)>c\left(H^{\prime \prime}\right) \Leftrightarrow C^{\prime}<C^{\prime \prime}, \quad \text { if } g^{\prime \prime}(x)<0 \text { on }[k, k+n-1] .
$$

Both of the intersection points of $H^{\prime}$ and $H^{\prime \prime}$ belong to $(k, k+n-1)$, so

$$
\begin{aligned}
& \left(x_{1}-B^{\prime}\right) \cdot\left(x_{2}-B^{\prime}\right)>0 \Leftrightarrow \quad-\frac{A^{\prime} \triangle B^{\prime}}{C^{\prime}-C^{\prime \prime}}>0, \\
& \left(x_{1}-B^{\prime}\right)+\left(x_{2}-B^{\prime}\right)>0 \Leftrightarrow \triangle B^{\prime}+\frac{\triangle A^{\prime}}{C^{\prime}-C^{\prime \prime}}>0,
\end{aligned}
$$


which implies

$$
\begin{gathered}
\triangle B^{\prime}<0 \text { and } \triangle A^{\prime}>0, \quad \text { if } g^{\prime \prime}(x)>0 \\
\triangle B^{\prime}>0 \text { and } \triangle A^{\prime}<0, \quad \text { if } g^{\prime \prime}(x)<0
\end{gathered}
$$$$
\text { and }
$$

Finally, here is the recovering algorithm for the digital hyperbola segment from its proposed $(k, n, a, b, c)$-code:

\section{Algorithm HYPERBOLA $(k, n, a, b, c, H)$}

Input: Numbers $k$ (the abscissa of the left endpoint of the segment), $n$ (length of the segment), $a, b$ and $c$.

Output: Hyperbola $H: y=\frac{A}{x-B}+C$, which satisfies $a(H)=a, b(H)=b$ and $c(H)=c$, i.e. which is corresponded to the coded digital segment.

Step 1: Choose the coefficient $B<k$;

$$
\operatorname{Set} \_a b(k, n, B, a, b, H) \text {; }
$$

Step 2: $\operatorname{IF}(c(H) \neq c)$

$\{$ choose the next $B$ by binary searching; $(*$ Lemma $6 *)$;

$\operatorname{Set} a b(k, n, B, a, b, H)\}$.

The procedure will be terminated when the solution (one of the hyperbolas corresponding to the given code) is found. If the initial lower bound for $B$ is given, the algorithm can be used for solving the recognition problem as well, since it will be shown that the solution, if exists, should be reached before the width of the interval containing $\beta$ becomes less then $\frac{1}{4 m^{4}}$.

In the end, complexity of the algorithm HYPERBOLA will be discussed.

Lemma 7 Every digital hyperbola segment can be obtained as the digitization of the hyperbola of the form $y=\frac{\alpha-\varepsilon_{\alpha}}{x-\left(\beta-\varepsilon_{\beta}\right)}+\gamma_{1}$, where $\alpha$ and $\beta$ are the coefficients of the hyperbola $y=\frac{\alpha}{x-\beta}+\gamma$ passing through three digital points, and $\varepsilon_{\alpha}$ and $\varepsilon_{\beta}$ are positive, small enough numbers.

Proof. Consider a hyperbola $h: y=\frac{a}{x-b}+c$.

By translating $h$ downward until it reaches the nearest digital point, $\left(x_{\gamma}, y_{\gamma}\right)$, we get hyperbola $h_{1}: y=\frac{a}{x-b}+\gamma$. By changing parameter $b$ till the next nearest point, $\left(x_{\beta}, y_{\beta}\right)$, is reached, we get hyperbola $h_{2}: y=\frac{a}{x-\beta}+\gamma$. Finally, changing the parameter $a$ and reaching the third digital point $\left(x_{\alpha}, y_{\alpha}\right)$ leads to the hyperbola $h_{3}: y=\frac{\alpha}{x-\beta}+\gamma$.

So, $h_{3}$ passes through three digital points and $y=\frac{\alpha-\varepsilon_{\alpha}}{x-\left(\beta-\varepsilon_{\beta}\right)}+\gamma_{1}$ is determined by the points $\left(x_{\alpha} \pm \varepsilon, y_{\alpha} \pm \varepsilon\right),\left(x_{\beta} \pm \varepsilon, y_{\beta} \pm \varepsilon\right)$ and $\left(x_{\gamma} \pm \varepsilon, y_{\gamma} \pm \varepsilon\right)$, where $\varepsilon$ is positive, small enough number, and sign " + " is taken for those of the points $\left(x_{\alpha}, y_{\alpha}\right),\left(x_{\beta}, y_{\beta}\right),\left(x_{\gamma}, y_{\gamma}\right)$ which are elements of the digital segment, else "-" is taken. Obviously, $y=\frac{\alpha-\varepsilon_{\alpha}}{x-\left(\beta-\varepsilon_{\beta}\right)}+\gamma_{1}$ and $h$ have the same digitization.

Theorem 2 Any digital hyperbola segment, corresponding to the hyperbola of the form $y=\frac{\alpha}{x-\beta}+\gamma$ and presented on the $m \times m$ integer grid, represented by its $(k, n, a, b, c)$-code, can be recovered in $\mathcal{O}\left(m \cdot(\log (m+|\beta|))^{2}\right)$ time. 
Proof. The coefficients $\alpha$ and $\beta$ of the hyperbola $y=\frac{\alpha}{x-\beta}+\gamma$ passing through three given points $\left(x_{1}, y_{1}\right),\left(x_{2}, y_{2}\right),\left(x_{3}, y_{3}\right)$ are determined as follows:

$$
\begin{gathered}
\alpha=\frac{\left(x_{1}-x_{2}\right)\left(x_{1}-x_{3}\right)\left(x_{3}-x_{2}\right)\left(y_{2}-y_{1}\right)\left(y_{2}-y_{3}\right)\left(y_{3}-y_{1}\right)}{\left(\left(y_{2}-y_{1}\right)\left(x_{1}-x_{3}\right)-\left(y_{3}-y_{1}\right)\left(x_{1}-x_{2}\right)\right)^{2}}, \\
\beta=\frac{x_{2}\left(y_{2}-y_{1}\right)\left(x_{1}-x_{3}\right)-x_{3}\left(y_{3}-y_{1}\right)\left(x_{1}-x_{2}\right)}{\left(y_{2}-y_{1}\right)\left(x_{1}-x_{3}\right)-\left(y_{3}-y_{1}\right)\left(x_{1}-x_{2}\right)} .
\end{gathered}
$$

So, for the difference between different coefficients $\alpha_{1}$ and $\alpha_{2}$, and analogously, $\beta_{1}$ and $\beta_{2}$, of the hyperbolas $y=\frac{\alpha_{1}}{x-\beta_{1}}+\gamma_{1}$ and $y=\frac{\alpha_{2}}{x-\beta_{2}}+\gamma_{2}$, each passing through three points of a grid of size $m \times m$, we have

$$
\left|\alpha_{1}-\alpha_{2}\right|>\frac{1}{16 m^{8}}, \quad \text { and } \quad\left|\beta_{1}-\beta_{2}\right|>\frac{1}{4 m^{4}} .
$$

The width of $I(\alpha)$ is (see Lemma 4 )

$$
\begin{gathered}
\frac{2 n(2 k+n-1)}{\sum_{i=1}^{n} \frac{n+1-2 i}{k+i-1-\beta}}<4(2 k+n-1)(k+n-1-\beta)^{2}, \\
\sum_{i=1}^{n} \frac{n+1-2 i}{k+i-1-\beta}>\frac{n}{2(k+n-1-\beta)^{2}} .
\end{gathered}
$$

since

Taking into account that $k$ and $n$ are at most equal to $m$, we have $I(\alpha)<$ $12 m(2 m+|\beta|)^{2}$, which implies that $\mathcal{O}(\log (m+|\beta|))$ iterations will be needed to obtain $I(\alpha)$ less then $\frac{1}{16 m^{8}}$.

For locating $\beta, \mathcal{O}(\log (m+|\beta|))$ iterations will be needed, too; $I(\beta)$ will be less then $\frac{1}{4 m^{4}}$ after that. With Lemma 3 , the statement follows.

Table 1. contains some numerical examples.

Table 1. The digital segment $H_{k}(h, n)$, obtained by digitizing $h: y=\frac{\alpha}{x-\beta}+\gamma$ on the interval $[k, k+n-1]$, is coded by $(k, n, a, b, c)$-code, where $g$ is chosen to be $x^{2}$. After applying the recovering procedure, the parameters $A, B, C$ of the hyperbola $h_{r e c}: y=\frac{A}{x-B}+C$, having the same corresponding digital segment on the observed interval, are obtained

\begin{tabular}{|r|r|r|c||r|r|r|}
\hline$\alpha$ & $\beta$ & $\gamma$ & $(k, n, a, b, c)$ & $A$ & $B$ & $C$ \\
\hline \hline \multirow{2}{*}{5.12} & \multirow{2}{*}{2.88} & \multirow{4}{*}{4.31} & $(3,7,80,350,1842)$ & 5.278417477 & 2.875 & 4.223145 \\
\cline { 4 - 7 } & & & $(4,42,179,4182,125968)$ & 4.529032519 & 3 & 4.393433 \\
\cline { 4 - 7 } & & $(45,27,108,6264,369864)$ & 0.890407522 & 44 & 4 \\
\hline \hline \multirow{2}{*}{17.19} & \multirow{2}{*}{0.53} & \multirow{2}{*}{2.77} & $(1,4,36,77,209)$ & 10.758620689 & 0 & 4 \\
\cline { 3 - 7 } & & & $(10,22,70,1399,30927)$ & 0.744398733 & 9 & 3.83251 \\
\cline { 3 - 7 } & & & $(25,67,184,10247,633817)$ & 0.702846342 & 24 & 2.98608 \\
\hline
\end{tabular}




\section{Comments and Conclusion}

Our research has been motivated by the fact that the conic sections are the most often appearing digital curves in practice of computer vision and image processing. For all of them the different solutions of the coding problem are known, and for most of them either a recovering procedure or the parameter estimation of the coded digital curve can be found in the literature. But as far as the digital segment corresponding to the general hyperbola is considered, only the problem of representation can be solved, by applying the general result developed in [8]. The proposed code consists of five parameters. Four of them are strictly determined, while the fifth can be chosen according to the practical reasons, since it depends on an arbitrary strictly convex (concave) function $g(x)$, having the second derivative on the observed interval. In the previous sections the recovering procedure for the digital hyperbola segments, represented by that code, is described. If the particular function $g(x)$ is noted, the recovering algorithm can be applied for any choice of $g(x)$. In practice, if $y=\frac{\alpha}{x-\beta}+\gamma$ is coded on the interval $[k, k+n-1]$, the information about the sign of $\alpha$ and $(k-\beta)$ is supposed to be included in the code, as well; the conclusion analogous to those of Lemma 5 and Lemma 6 , permitting binary searching, can be derived for any of the cases. The result is obtained in $\mathcal{O}\left(m \cdot(\log (m+|\beta|))^{2}\right)$ time.

The main contribution of the paper is that it gives a complete and efficient solution to the representation and the reconstruction problem connected with one class of the digital images. The proposed code is simple, uniquely determined, recoverable and optimal, while the recovering algorithm enables the exact and fast reconstruction of the digital segment. For comparing, it might be noted that the algorithm for the exact reconstruction of the digital ellipse (circle) does not exist, even though the efficient coding scheme (based on the similar concept as proposed here) enables very efficient estimation of the parameters of the original object [10]. So the idea for the further research could be to find a constant time approximate reconstruction for the relevant parameters of the hyperbola from the code of its digital segment.

\section{References}

1. Blum, M., Floyd, R. W., Pratt, V., Rivest, R. L., Tarjan, R. E.: Time bounds for selection. J. Comput. System Sci. 7(4). (1973) 448-461 164

2. Dorst, L., Smeulders, A. W. M.: Discrete representation of straight lines. IEEE Trans. Pattern Analysis and Machine Intelligence, Vol. 6. (1984) 450-463 159

3. Kim, C. E.: Digital disks. IEEE Trans. Pattern Analysis and Machine Intelligence, Vol. 6. (1984) 372-374

4. Lindenbaum, M., Koplowitz, J.: A new parametrization of digital straight lines. IEEE Trans. Pattern Analysis and Machine Intelligence, Vol. 13. No.4. (1991) 847852

5. Melter, R. A., Stojmenović, I., Žunić, J.: A new characterization of digital lines by least square fits. Pattern Recognition Letters, Vol. 14. (1993) 83-88 
6. Woring, M., Smeulders, A. W. M.: Digitized Circular Arcs: Characterization and Parameter Estimation. IEEE Trans. Pattern Analysis and Machine Intelligence, Vol. 17. (1995) 587-597

7. Žunić, J., Koplowitz, J.: A representation of digital parabolas by least square fits. SPIE Proc., Vol. 2356. (1994) 71-78 159

8. Žunić, J.: A coding scheme for certain sets of digital curves. Pattern Recognition Letters, Vol. 16. (1995) 97-104 159, 160, 161, 169

9. Žunić, J.: A Representation of Digital Hyperbolas $y=\frac{1}{x} \alpha+\beta$. Pattern Recognition Letters, Vol. 17. (1996) 975-983 159

10. Žunić J., Sladoje, N.: Efficiency of Characterizing Ellipses and Ellipsoids by Discrete Moments. IEEE Trans. Pattern Analysis and Machine Intelligence, Vol. 22. (2000) 407-414 159, 169 